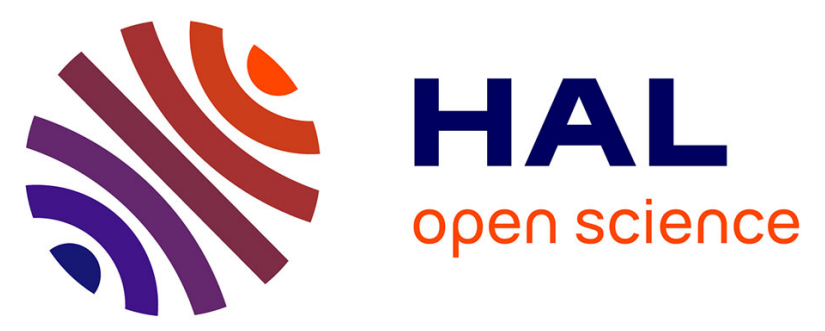

\title{
Heat transfer measurements of a nanoscale hot-wire in supersonic flow
}

Katherine Kokmanian, Diogo C. Barros, Marcus Hultmark, Pierre Dupont

\section{To cite this version:}

Katherine Kokmanian, Diogo C. Barros, Marcus Hultmark, Pierre Dupont. Heat transfer measurements of a nanoscale hot-wire in supersonic flow. Experiments in Fluids, 2021, 62 (8), 10.1007/s00348021-03259-8 . hal-03411547

\section{HAL Id: hal-03411547 https://hal-amu.archives-ouvertes.fr/hal-03411547}

Submitted on 2 Nov 2021

HAL is a multi-disciplinary open access archive for the deposit and dissemination of scientific research documents, whether they are published or not. The documents may come from teaching and research institutions in France or abroad, or from public or private research centers.
L'archive ouverte pluridisciplinaire HAL, est destinée au dépôt et à la diffusion de documents scientifiques de niveau recherche, publiés ou non, émanant des établissements d'enseignement et de recherche français ou étrangers, des laboratoires publics ou privés. 


\title{
Heat transfer measurements of a nanoscale hot-wire in supersonic flow
}

\author{
Katherine Kokmanian · Diogo C. Barros · Marcus Hultmark · Pierre Dupont
}

Received: date / Accepted: date

\begin{abstract}
Upon its development and initial characterization, the supersonic variant of the nanoscale thermal anemometry probe (S-NSTAP) was deployed in a supersonic wind tunnel facility, where both freestream and boundary layer measurements were obtained at $M_{\infty}=2$. The low operating stagnation pressures generated reliable data, where the effects of Reynolds number, Mach number and overheat ratio on the sensor's heat transfer were investigated in detail. The performance of the S-NSTAP was also compared to that of a conventional cylindrical hot-wire and the S-NSTAP was shown to exhibit unparalleled temporal resolution $(\sim 300$ $\mathrm{kHz}$ ). The mass flux sensitivity coefficient of both hot-wires was further computed and appeared to vary between probes, yielding a coefficient twice as large for the conventional probe than for the S-NSTAP. The experimental data obtained from both hot-wires were also compared, via spectral analysis and turbulence statistics, to the results of a numerically modelled turbulent boundary layer.
\end{abstract}

Keywords Hot-wire anemometry · Nanoscale sensors · Supersonic flows

\section{Introduction}

Due to the short time scales ever so present in supersonic flows, hot-wires have traditionally been the prevailing method for obtaining highly resolved turbulence statistics. Standard hot-wires consist of a thin electrically conductive filament heated via Joule heating. Typical dimensions range between

Katherine Kokmanian, Marcus Hultmark

Mechanical and Aerospace Engineering Department

Princeton University, Princeton, USA

E-mail: kkokmanian@alumni.princeton.edu

Diogo C. Barros, Pierre Dupont

Aix Marseille Université, CNRS, IUSTI, Marseille, France

E-mail: diogo.camello-barros@univ-amu.fr
0.5 and $5 \mu \mathrm{m}$ in diameter and between 0.5 and $2 \mathrm{~mm}$ in length (Tropea et al, 2007; Comte-Bellot, 1976). When placed in a flow of interest, the change in the hot-wire's heat transfer can be correlated to a change in wire resistance if operated in a constant current anemometry (CCA) circuit, or a change in current if operated in constant voltage (CVA) or temperature (CTA) anemometry circuits (Tropea et al, 2007).

In recent years, the advancement in semiconductor manufacturing techniques allowed for the development of nanoscale thermal anemometry probes (NSTAP). Results presented in Bailey et al (2010) confirmed the proper functioning of the NSTAP, while showcasing its superior spatial and temporal resolution. Given its smaller size and faster response compared to a conventional hot-wire, its application in highspeed flows is particularly alluring. The NSTAP was therefore redesigned and tested for this very purpose (Kokmanian et al, 2019; Kokmanian, 2020). However, in order to completely comprehend its output, a thorough investigation of the governing heat transfer from hot-wires in high-speed flows must be undertaken.

\subsection{Heat transfer from hot-wires in high-speed flow}

Assuming a steady flow, the convective heat loss, $H$, can be normalized using $N u=\frac{h \ell_{c}}{k}$ :

$N u=\frac{H}{k \frac{A}{\ell_{c}}\left(T_{w}-T_{e}\right)}$.

Here, $h$ denotes the heat transfer coefficient, $k$ denotes the thermal conductivity of the fluid, $\ell_{c}$ is a characteristic length scale of the wire while $A$ is the surface area of the sensing element. For a conventional cylindrical hot-wire, its diameter, $d$, represents the characteristic length scale and its surface area is simply $A=\pi d \ell$. However, considering a thin 
sensing element of ribbon-like geometry, both its thickness, $t$, and its width, $w$, can potentially be the governing length scale. As for the surface area, it is rather $A=2 w \ell$, where $\ell$ is the length of the filament. The temperature difference in Equation 1, also denoted as $\Delta T=T_{w}-T_{e}$, represents the difference between the temperature of the heated wire, $T_{w}$, and the recovery temperature, $T_{e}$, the latter of which is namely the equilibrium temperature of the unheated wire for a given aerodynamic condition. In practice, the convective heat loss is balanced, and hence measured, by the electrical supply: $H=E_{w}^{2} / R_{w}=R_{w} i^{2}$. Here, $E_{w}, R_{w}$ and $i$ are the wire's voltage, heated resistance and traversing current respectively.

In his seminal analysis of hot-wire anemometry in supersonic flows, Kovásznay (1950) showed through dimensional analysis that the Nusselt number, $\mathrm{Nu}$, is a function of the following five parameters:

$N u=N u\left(\operatorname{Re}, \operatorname{Pr}, M, \tau, \frac{\ell}{\ell_{c}}\right)$.

The Reynolds number, $R e=\frac{\rho u \ell_{c}}{\mu}$, is a function of density, $\rho$, velocity, $u$, and dynamic viscosity, $\mu$, while the Prandtl number, $\operatorname{Pr}=\frac{\mu c_{p}}{k}$, depends on $\mu, k$ as well as the specific heat at constant pressure, $c_{p}$. The Mach number, $M$, is defined as the ratio between the flow velocity, $u$, and the speed of sound, $a$, while the overheat ratio, $\tau=\frac{\Delta T}{T_{0}}$, is the ratio between $\Delta T$ and the stagnation temperature, $T_{0}$. Lastly, $\frac{\ell}{\ell_{c}}$ denotes the aspect ratio of the wire filament and is constant for a given sensor. Although the fluid properties $\mu$ and $k$ vary with temperature, they are typically evaluated at the stagnation conditions since the shock generated upstream of the wire increases the static temperature of the flow downstream of the shock close to the value of $T_{0}$ (Kovásznay, 1950; Kovásznay and Törmarck, 1950).

For a given wire geometry and nearly constant $\operatorname{Pr}$ conditions, the relation can be simplified to:

$N u=N u(\operatorname{Re}, M, \tau)$.

Laufer and McClellan (1956) investigated the effect of $M$ on $N u$ and found $N u$ to be insensitive to $M$ for $R e>20$ and $1.3<M<4.5$. Extensive measurements performed by Barre et al (1992) confirmed this trend for $M>1.3$. In particular, for this Mach number range, the experiments from Kovásznay (1950) and Laufer and McClellan (1956) clearly demonstrated a power-law relationship of the following form:

$N u=f(\tau)+g(\tau) R e^{n}$ for $M>1.3$,

where $f$ and $g$ are non-dimensional functions solely dependent on $\tau$, and $\ell_{c}$ is selected as the diameter, $d$, for cylindrical hot-wires (Kovásznay, 1950; Smits et al, 1983). As for the exponent, $n$, both Kovásznay and Törmarck (1950) and Laufer and McClellan (1956) investigated the behavior of conventional hot-wires in supersonic flows and found that $n=0.5$ agreed well with the entirety of their data for $R e>20$. A square root dependence on $R e$ leads to the seminal King's law (King, 1914). Later, Smits et al (1983) placed a $5 \mu \mathrm{m}$ hot-wire in a $M=2.9$ flow and rather found that $n=0.55$ best fit their calibration data.

Unlike $\mu$, the variation in $\rho$ cannot be neglected in the study of hot-wire anemometry in supersonic flows; its effect cannot be decoupled to that of $u$. That being said, hot-wires measure the mass flux, $\rho u$. Therefore, when deploying a hotwire in supersonic flow, a calibration is sought between $\mathrm{Nu}$ and $R e$, or, more practically, between $E_{w}$ and $\rho u$.

It is important to note that as $M$ increases, or equivalently as $R e$ decreases, rarefied gas effects become significant. The non-dimensional number of importance is known as the Knudsen number, $K n$, generally defined as:

$K n=\frac{\lambda}{\ell_{c}}$,

or, making use of kinetic theory of gases (Dewey Jr., 1965; Gatski and Bonnet, 2009):

$K n=\sqrt{\frac{\pi \gamma}{2}} \frac{M}{R e}$.

In the above equations, $\lambda$ is the mean free path of the fluid, $\ell_{c}$ remains the characteristic length scale of the wire and $\gamma$ is the ratio of specific heats. Stalder et al (1951) found that, for large $K n$, the governing heat transfer becomes linear, i.e. the exponent in Equation 4 equals unity. Baldwin et al (1960) compiled supersonic heat transfer data of transverse cylindrical hot-wires in a rarefied air stream and clearly identified the linear $N u-R e$ relationship. Dewey Jr. (1965) later compiled hot-wire data obtained in various conditions and observed a smooth transition in exponent from sensor operation in continuum flow, where $n=0.5$, to operation in free-molecule flow, where $n=1$. A similar compilation of experimental results is shown in Figure 1, where data from Kovásznay (1950), Stalder et al (1952), Laufer and McClellan (1956), Dewey Jr. (1961) and Weltmann and Kuhns (1960) are visually depicted. A clear shift in slope can be observed. It must be noted that all data sets included in Figure 1 were obtained with conventional cylindrical hot-wires.

\subsection{The nanoscale thermal anemometry probe}

With the advent of micro-electro-mechanical systems, the nanoscale thermal anemometry probe (NSTAP) was developed in response to the need for highly resolved turbulence measurements (Bailey et al, 2010; Fan et al, 2015). This miniature silicon-based hot-wire contains a nanoscopic layer of platinum which comprises the sensing element. The temperature coefficient of resistance of the platinum layer was 


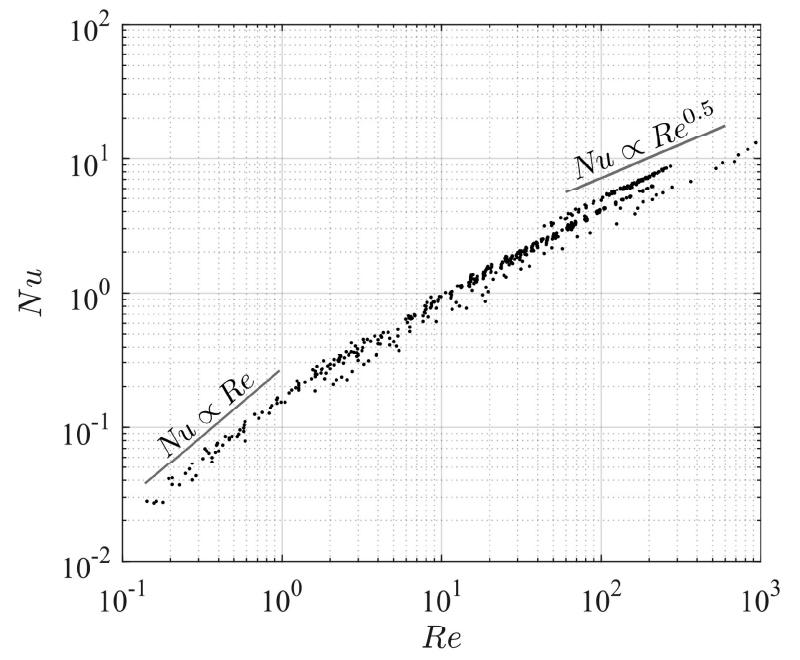

Fig. 1: Compilation of heat transfer measurements in supersonic flows. The data points were extracted from the experiments reported by Kovásznay (1950), Stalder et al (1952), Laufer and McClellan (1956), Dewey Jr. (1961) and Weltmann and Kuhns (1960). The reported Mach numbers lie within the range $1.15<M<5.8$ while the overheat ratio spans $0<\tau<1$.

experimentally found to be $\alpha=0.0021 \mathrm{~K}^{-1}$. Due to the deposition process of the metallic layer, the cross section of the sensing element is rectangular as opposed to being circular.

The NSTAP is fabricated in Princeton University's clean room, providing complete control of its design and manufacturing. Due to its high spatial and temporal resolution, the NSTAP was recently altered in order to operate efficiently in high-speed flows (Kokmanian et al, 2019; Kokmanian, 2020). Figure 2 illustrates the supersonic variant of the NSTAP, henceforth denoted as the S-NSTAP. In Figures $2(a, b)$, the sensor is soldered onto prongs, showcasing the entirety of the structure. Figures $2(\mathrm{c}, \mathrm{d})$ rather focus on the two-dimensional pattern of the metallic layer of the S-NSTAP. The heated ribbon, at the very tip of the structure, is $30 \mu \mathrm{m}$ long, $2 \mu \mathrm{m}$ wide and approximately $400 \mathrm{~nm}$ thick. The silicon supports at each end of the sensing element are tapered in order to maintain an aerodynamic shape and extract near-wall measurements, while the silicon bridge downstream of the wire is intended to weaken wire vibration. Additional information regarding the design modifications can be found in Kokmanian et al (2019).

Given the difference in cross section compared to a conventional hot-wire, selecting the S-NSTAP's characteristic length scale for the calculation of both $R e$ and $K n$ is not obvious. For instance, selecting the width of the sensing element as the characteristic length scale yields $K n_{w t}=0.98$ for $M=2$ and $R e_{w t}=3$, while $K n_{t}=4.9$ if the thickness were selected instead. Since both values are greater than the
$K n=0.001$ threshold for continuum flow (Rapp, 2017), rarefied gas effects cannot be excluded and the appropriate exponent for the $\mathrm{Nu}-\mathrm{Re}$ calibration is not known a priori. The goal of this study is to provide a comprehensive characterization of the S-NSTAP's heat transfer in supersonic flows. This is done by isolating the effect of $\operatorname{Re}, M$ and $\tau$ on $N u$ and computing the mass flux sensitivity coefficient of the sensor. In addition, the spectral response of the S-NSTAP is compared to that of a cylindrical hot-wire based on measurements across a turbulent boundary layer with free-stream Mach number $M_{\infty}=2$. Data from both hot-wires are also compared to results from a numerical simulation.

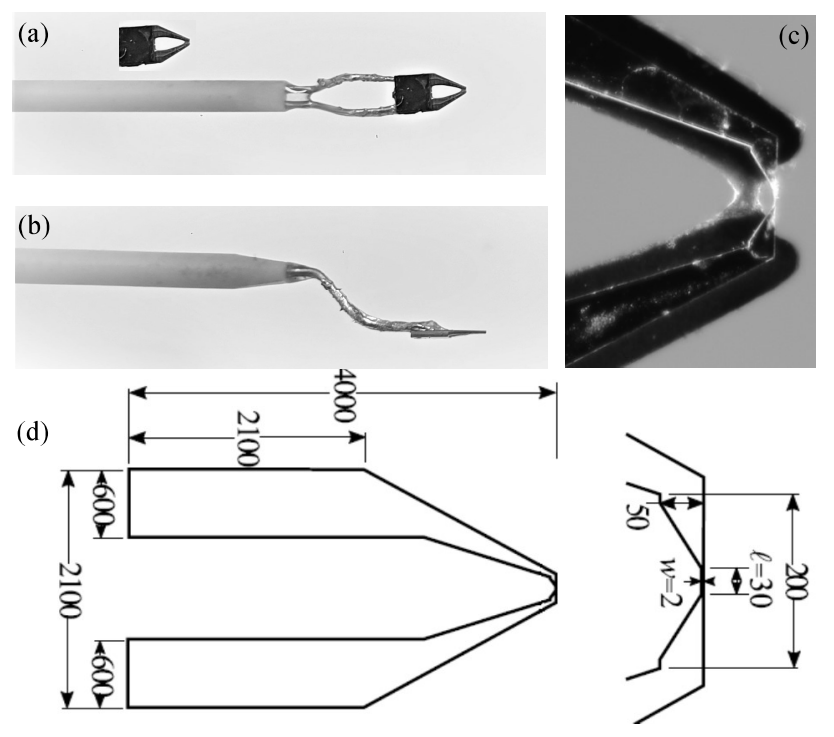

Fig. 2: Top (a) and side (b) views of the S-NSTAP soldered onto copper-plated stainless steel prongs for mounting purposes (flow, in this case, is from right to left); (c) microscope image showcasing the layer of platinum, under which lies a layer of silicon. This image focuses on the tip of the sensor where the silicon bridge, downstream of the sensing element, is also captured; (d) dimensions of the platinum layer, adapted from Kokmanian et al (2019). The freestanding wire, located at the very tip and highlighted in the zoomed-in image, is $30 \mu \mathrm{m}$ long, $2 \mu \mathrm{m}$ wide and $400 \mathrm{~nm}$ thick. All displayed dimensions are in micrometers.

\section{Experimental setup and methodology}

All experiments were performed in the supersonic wind tunnel facility located at the Institut Universitaire des Systèmes Thermiques et Industriels (IUSTI) in Marseille, France. This facility is ideally built for hot-wire measurements, given its low freestream turbulence levels. Furthermore, its low operating stagnation pressure, ranging from 0.15 to 0.9 atm, 
generates low loading on the sensor and enhances its survivability. This facility also has the capability of generating supersonic flow continuously while maintaining stable temperature and pressure. It is equipped with total pressure and total temperature sensors, the readings of which are recorded during sensor operation. Three pieces of tape are secured along the spanwise direction upstream of the throat in order to trip the boundary layers, present on the top and bottom walls of the test section, to a turbulent state. The test section is $105 \mathrm{~mm}$ high and $170 \mathrm{~mm}$ wide. A traverse system combined with an external scope are used to ensure submillimeter accuracy $(<0.1 \mathrm{~mm})$ in the positioning of the hot-wire.

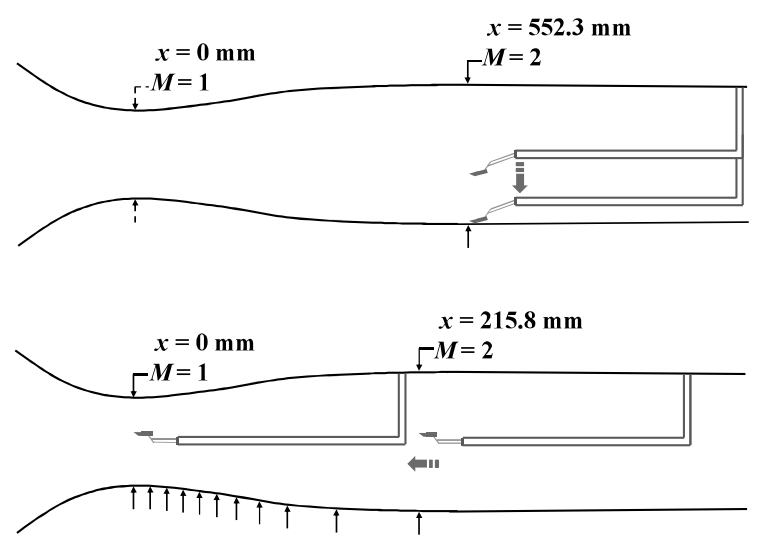

Fig. 3: Schematics of the converging-diverging nozzle followed by the $M=2$ test section, showcasing where the experimental measurements were taken (not to scale). Both the Reynolds number calibration and the boundary layer profile were obtained in the $M=2$ test section (top) while the S-NSTAP was traversed between the throat and the test section for Mach number calibration (bottom). The solid arrows mark the various measurement positions.

Multiple tests were considered in order to accurately investigate the effect of the Reynolds number, the Mach number as well as the overheat ratio. The variation in $R e$ was achieved by changing the stagnation pressure, while $M$ was varied by altering the streamwise sensor location within the converging-diverging nozzle upstream of the test section. The effect of $\tau$ was investigated by modifying the external resistance in the Wheatstone bridge of the anemometer. Figure 3 displays the experimental configurations for both the Re and $M$ calibrations.

For all tests, the hot-wires were operated in a constanttemperature anemometry mode (CTA), where the Dantec StreamLine CTA 90C10 module was used. The bridge configuration was set to 1:1 for all S-NSTAP measurements as well as for measurements performed with a $d=5 \mu \mathrm{m}$ and $\ell=0.9 \mathrm{~mm}$ conventional cylindrical wire. The correspond- ing non-dimensional lengths were $\ell^{+}=2.4$ and $\ell^{+}=72$ respectively, where the + subscript represents a scaling in viscous units (see the boundary layer results in \$4).

In addition, the S-NSTAP was further characterized by obtaining mass flux profiles along a $M_{\infty}=2$ turbulent boundary layer (the measurement location is also shown in Figure 3) at two distinct total pressures. The data collected with the S-NSTAP were compared to results obtained from the large eddy simulation (LES) of a canonical supersonic turbulent boundary layer described in Schreyer et al (2016). The parameters of the simulation were the following: $M_{\infty}=$ $2, p_{0 \infty}=0.4 \mathrm{~atm}, T_{0 \infty}=293 \mathrm{~K}, u_{\tau}=21.2 \mathrm{~m} / \mathrm{s}, R e_{\tau}=\delta u_{\tau} / v_{\text {wall }}=$ 893 and $R e_{\theta}=\theta u_{\infty} / v_{\infty}=5150$. Here, $\delta$ denotes the boundary layer thickness, $u_{\tau}$ represents the friction velocity, $v$ denotes the kinematic viscosity, while $\theta$ represents the momentum thickness. The high grid resolution in viscous units, namely $\Delta x^{+} \approx 30$ in the streamwise direction and $\Delta z^{+} \approx 12$ in the spanwise direction, allowed for the simulation to be considered wall-resolved. The selective mixed-scale model was selected as the subgrid-scale model for this simulation. The numerical results were obtained $55 \mathrm{~cm}$ downstream of the nozzle throat; the corresponding boundary layer thickness measured from the velocity profile equaled $\delta=11.16 \mathrm{~mm}$.

\section{Heat transfer measurements}

\subsection{Mass flux calibration}

In order to investigate the supersonic heat transfer law at very small scales, the S-NSTAP was initially placed in the freestream at $M=2$. The stagnation pressure was incrementally increased from 0.19 to 0.87 atm in order to vary the Reynolds number, while keeping all other parameters fixed. The objective was to investigate the heat transfer behavior of the S-NSTAP over a reasonably large mass flux range corresponding to low Reynolds numbers. As observed in Figure 4, a convincing $n=0.5$ fit both the $N u-R e_{w t}$ and the $\overline{E_{w}^{2}} / R_{w}-\overline{\rho u}$ calibrations well. The difference between the current results and the ones presented in Kokmanian et al (2019) can be attributed to the wider range of $R e_{w t}$. It must be noted that the subscript $w t$ denotes that the width of the sensing element was selected as the characteristic length scale, while variables with the subscript $w$ rather designate that they were evaluated at the heated wire condition.

It is also of interest to remark that the values of $N u$ for the S-NSTAP are significantly larger than the normalized heat loss of conventional hot-wires presented in Figure 1. This increase in $N u$ is primarily due to an artificial increase in resistance; although $R_{w}$ is defined as the wire resistance, the total sensor resistance is instead measured for practical purposes. Given that the probe body has a certain resistance, this should be subtracted from the total resistance in order 
to obtain the exact resistance of the sensing element, i.e. $R_{w}=R_{\text {total }}-R_{\text {body }}$. However, since the exact value of $R_{b o d y}$ is unknown, it was lumped into $R_{w}$. This is not expected to affect the obtained measurements, given that the significantly larger surface area of the probe body does not cause a large increase in temperature. The probe body resistance is not expected to vary with mass flux, simply causing an offset in the calibration. Future work will focus on determining the exact wire resistance.
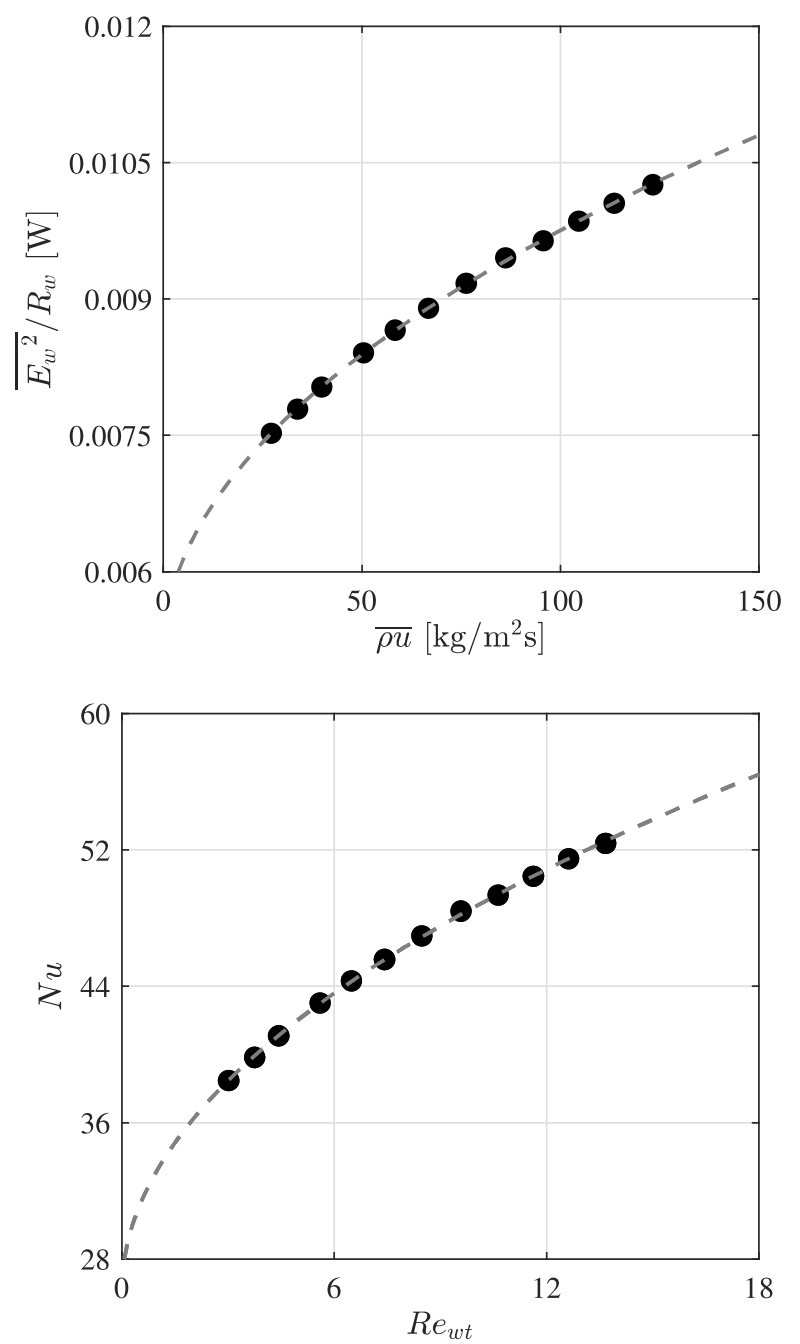

Fig. 4: Top: $\overline{E_{w}^{2}} / R_{w}-\overline{\rho u}$ calibration with a fit of $\overline{E_{w}^{2}} / R_{w}=$ $0.0051+0.00047 \sqrt{\overline{\rho u}}$ where $R_{w}=28.5 \Omega$. Bottom: $N u-$ $R e_{w t}$ calibration with a fit of $N u=26.05+7.16 \sqrt{R e_{w t}}$. Both fits agree well with the experimental data $\left(R^{2}=0.9998\right.$ and 0.9997 respectively), collected at $M=2$ and $\tau=0.44$.

Upon extraction of the $N u-R e$ calibration, boundary layer data were collected with the S-NSTAP at $p_{0 \infty}=0.5$ and $0.8 \mathrm{~atm}$ and were subsequently converted to mass flux (assuming no Mach number and no total temperature effects). $R e_{\theta}$ was measured in a previous campaign and was found to be $R e_{\theta}=4850$ at $M_{\infty}=2$ and $p_{0 \infty}=0.4$ atm (Schreyer et al, 2016).

Data were recorded throughout the boundary layer with an increment of $y / \delta \approx 0.1$. The wall-normal distance was normalized by the boundary layer thickness defined as the location where $\overline{\rho u}=0.99(\overline{\rho u})_{\infty}$. Unsurprisingly, the value of $\delta$ varied between both data sets, where it equaled $\delta=$ $11.88 \mathrm{~mm}$ when $p_{0 \infty}=0.5 \mathrm{~atm}$ and $11.35 \mathrm{~mm}$ when $p_{0 \infty}=$ $0.8 \mathrm{~atm}$. A standard cylindrical wire $(d=5 \mu \mathrm{m}$ and $\ell=$ $0.9 \mathrm{~mm}$ ) was also used as an experimental benchmark, where a $N u-R e$ calibration was extracted at $M=2$ in the freestream, followed by a boundary layer measurement campaign at $p_{0 \infty}=$ $0.5 \mathrm{~atm}$.

Figure 5 depicts the normalized mass flux profiles. Good agreement can be found between the experimental and numerical results for $y / \delta>0.5$. Closer to the wall, the SNSTAP data appear to deviate slightly from the other two data sets, with a maximum of a $10 \%$ undershoot compared to the LES data set. Since the data were only calibrated in $R e$, the possibility of $M$ or $T_{0}$ variation influencing the data is possible. However, the transonic $M=1.3$ threshold for $M$ independence, suggested by Laufer and McClellan (1956) and confirmed by Barre et al (1992), occurs at $y / \delta=0.18$. The closest experimental data point collected with the hot-wires was at $y / \delta \approx 0.086$, indicating that previously reported $M$ number effects can only influence the point nearest to the wall. As for the effect of $T_{0}, T_{e}$ was recorded during the collection of data and appeared to stay constant throughout the boundary layer. The recovery ratio, $\eta=\frac{T_{e}}{T_{0}}$, has been found to range between 0.95 and 0.97 for a variety of supersonic flows and has been said to be $M$ independent in the continuum flow regime, where $K n \ll 1$ (Spina and McGinley, 1994). If the S-NSTAP operated in the continuum regime, then a constant $T_{e}$ and a relatively constant $\eta$ would imply a constant $T_{0}$. However, given the low local $R e$ and high associated $K n$ seen by the S-NSTAP, this is not necessarily valid.

\subsection{Sensitivity analysis}

In the investigation of supersonic turbulent flows, in particular boundary layers, the voltage fluctuations for a given wire can most generally be expressed in terms of mass flux, total temperature and Mach number variations:

$$
\frac{e^{\prime}}{\overline{E_{w}}}=F_{\rho u} \frac{(\rho u)^{\prime}}{\overline{\rho u}}+F_{T_{0}} \frac{T_{0}^{\prime}}{\overline{T_{0}}}+F_{M} \frac{M^{\prime}}{\bar{M}},
$$

where $F_{\rho u}, F_{T_{0}}$ and $F_{M}$ represent the non-dimensional mass flux, total temperature and Mach number sensitivity coefficients, respectively. A detailed analysis of the sensitivity coefficients was presented by Morkovin (1956). 


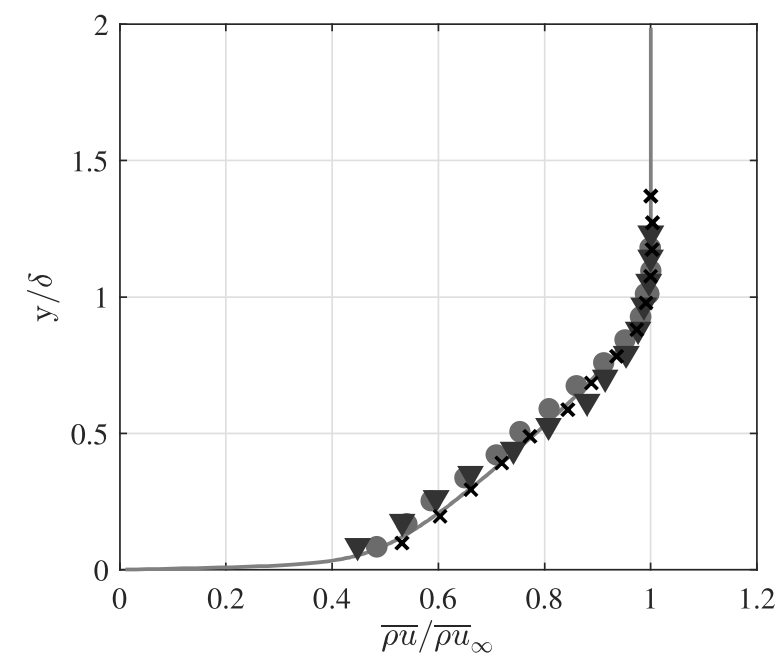

Fig. 5: Normalized mass flux profiles across the turbulent supersonic boundary layer. The S-NSTAP acquired data at two distinct stagnation pressures: $p_{0 \infty}=0.5 \mathrm{~atm}(\mathrm{O})$ and $p_{0 \infty}=0.8 \mathrm{~atm}(\nabla)$. Data from the conventional hot-wire are depicted in black $(\times)$, while the LES is depicted in grey.

The effect of $M$ can be encompassed in the other two sensitivity coefficients (Dupont and Debiève, 1992):

$$
\begin{aligned}
\frac{e^{\prime}}{\overline{E_{w}}}= & \left(F_{\rho u}+F_{M} \frac{1+\frac{\gamma-1}{2} M^{2}}{1+(\gamma-1) M^{2}}\right) \frac{(\rho u)^{\prime}}{\overline{\rho u}}+ \\
& \left(F_{T_{0}}+\frac{F_{M}}{2} \frac{1+\frac{\gamma-1}{2} M^{2}}{1+(\gamma-1) M^{2}}\right) \frac{T_{0}^{\prime}}{\overline{T_{0}}},
\end{aligned}
$$

where the expression in the first set of brackets, encompassing both $F_{\rho u}$ and $F_{M}$, is named $F_{1}$ for simplicity. In order to extract the dependence on $\rho u$, the $E_{w}^{2}-\rho u$ calibration, obtained at $M=2$, was directly applied to the boundary layer signals collected both at $p_{0 \infty}=0.5$ and 0.8 atm. Shown in Figure 6 is the normalized standard deviation of the voltage fluctuations, plotted against that of the mass flux fluctuations. Albeit at distinct $p_{0 \infty}$, both data sets collapse on a line with a slope of $F_{1}=0.12$. A downward hook appears at the maximum turbulence level (locations closest to the wall). Repeating this process for the conventional $5 \mu \mathrm{m}$ cylinder yields $F_{1}=0.25$, namely twice the S-NSTAP's mass flux sensitivity.

Of course, the values of $F_{1}$ obtained above depend on the calibration coefficients. Since the wire voltage was approximated by a law of the type $E_{w}^{2}=a_{1}+b_{1}(\rho u)^{n}$, the first order approximation of the voltage fluctuations implies:

$F_{\rho u}=\frac{n}{2}\left(\frac{1}{1+\frac{a_{1}}{b_{1}(\overline{\rho u})^{n}}}\right)$.

The calculation of $F_{\rho u}$ based on the calibration coefficients reported in Table 1 gives a systematic value for the

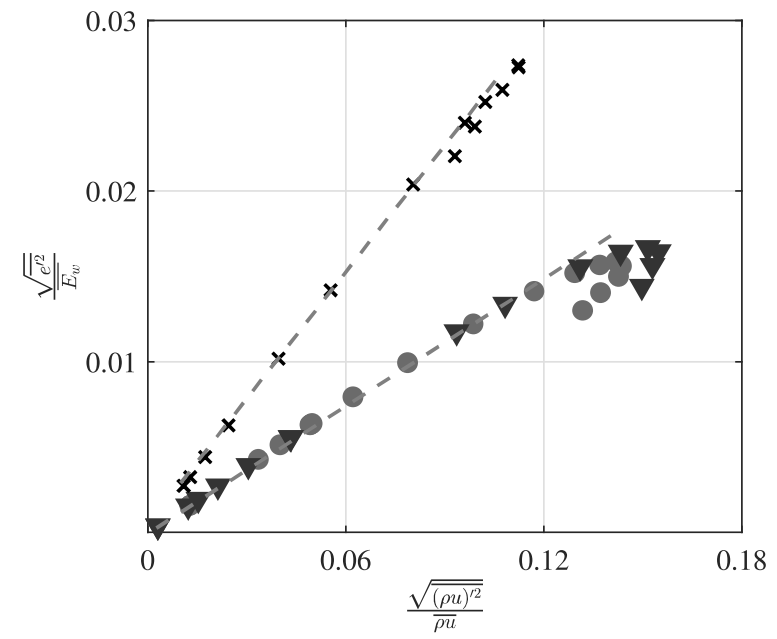

Fig. 6: Standard deviation of voltage fluctuations normalized by local mean wire voltage, plotted against the standard deviation of the mass flux fluctuations normalized by local mean mass flux. The mass flux sensitivity coefficient is determined by the slope of the curve and was found to be $F_{1}=0.12$ for both $p_{0 \infty}=0.5 \mathrm{~atm}(\mathrm{\circ})$ and $p_{0 \infty}=0.8 \mathrm{~atm}$ $(\nabla)$ data sets collected with the S-NSTAP, while it was rather $F_{1}=0.25$ for the conventional $5 \mu \mathrm{m}$ hot-wire at $p_{0 \infty}=0.5 \operatorname{atm}(\times)$.

Table 1: Calibration coefficients for both sensors.

\begin{tabular}{ccccccc} 
Sensor & $a_{1}\left[\mathrm{~V}^{2}\right]$ & $b_{1}\left[\frac{\mathrm{mV}^{2} \mathrm{~s}^{1 / 2}}{\mathrm{~kg}^{1 / 2}}\right]$ & $R_{w}[\Omega]$ & $\tau$ & $M$ & $n$ \\
\hline 5 $\mu \mathrm{m}$ & 0.00215 & 0.0154 & 4.45 & 0.73 & 2 & 0.5 \\
S-NSTAP & 0.145 & 0.0134 & 28.5 & 0.44 & 2 & 0.5 \\
\hline
\end{tabular}

$5 \mu \mathrm{m}$ cylindrical hot-wire of $F_{\rho u}=0.245 \pm 2 \%$ for any mass flux value within the calibration range (between 30 and 110 $\mathrm{kg} / \mathrm{m}^{2} \mathrm{~s}$ ). Comparing this to the results highlighted in Figure 6 would lead to the conclusion that $F_{1} \sim F_{\rho u}=0.245$ for most data points, thus confirming the absence of Mach number effects for $M>1.3$ (Barre et al, 1992). The same calculation performed for the S-NSTAP yields values of $F_{\rho u}$ between 0.084 and 0.123 in the same range of mass flux. The correction factor in the denominator of Equation 9 explains the leading order change with respect to the conventional cylindrical sensor. However, most of the initial boundary layer data points in Figure 6 (i.e. lower mass flux fluctuations corresponding to locations farther away from the wall) exhibit a linear trend pointing to $F_{1} \sim 0.12$, until the normalized standard deviation of the mass flux fluctuations reaches approximately 0.12 . Hence, the difference between $F_{\rho u}$ and $F_{1}$ is attributed to the effects of Mach number, either by the presence of $F_{M}$ in Equation 8 (not known a priori) or by a dependence of $a_{1}$ and $b_{1}$ on $M$. 
As for the sensitivity to total temperature, $T_{0}$ could unfortunately not be altered during a run. The overheat ratio, which in effect sets the temperature difference, $\Delta T$, was altered instead and the results are displayed in Section 3.4.

\subsection{Effect of Mach number}

Isolating the effect of $M$ on the heat transfer is challenging due to its coupling with other parameters. In practice, the Mach number was varied by traversing the sensor along the converging-diverging supersonic nozzle as depicted in Figure 3. However, this also leads to a variation in the mass flux $\rho u$ :

$\rho u=p_{0} \sqrt{\frac{\gamma}{R T_{0}}} M\left(1+\frac{\gamma-1}{2} M^{2}\right)^{-\frac{\gamma+1}{2(\gamma-1)}}$.

Keeping all other variables constant, a variation in $M$ leads to a change in $\rho u$. Therefore, the data collection was repeated for a range of stagnation pressures $p_{0}$ in order to obtain data at distinct $M$ but constant $\rho u$. Figure 7 displays the parameter space $\rho u-M$ covered by multiple stagnation pressures, making use of Equation 10. Data were collected at five different $p_{0}$, namely $0.23,0.30,0.41,0.50$ and $0.60 \mathrm{~atm}$, and at eleven downstream locations, depicted by the markers in the figure. For a given $p_{0}, M$ decreased while $\rho u$ increased as the hot-wire was brought closer to the nozzle's throat. The exact values of $M$ were obtained using a Pitot tube which traversed the converging-diverging nozzle. Figure 8 displays the mean wire voltage, $\overline{E_{w}}$, as a function of $M$, while Figure 9 depicts the variation between $\overline{E_{w}}$ and the local mean mass flux, $\rho u$. Interestingly, Figures 8 and 9 depict a non-monotonic variation of $\overline{E_{w}}$ throughout the converging-diverging nozzle. One cannot draw conclusions on the effect of $M$ on the mean voltage by merely analyzing Figure 8 since $\rho u$, and consequently $R e$, vary while traversing the hot-wire through the nozzle. Looking at Equation 10, although $p_{0}$ and $T_{0}$ remain invariant, a variation in $\rho u$ leads to a variation in $M$. Similarly, the dependence of $\overline{E_{w}}$ on $\rho u$, shown in Figure 9, varies from what was portrayed in Figure 4 due to the variation of $M$ throughout the data extraction.

In order to better understand the individual effect of $M$ on $\overline{E_{w}}$, smoothing splines were fitted in each $p_{0}$ data set and eight $\rho u$ values were selected and kept fixed while $M$ varied. These values are, in ascending order, 45, 50, 56, $70,83,90,96$ and $120 \mathrm{~kg} / \mathrm{m}^{2} \mathrm{~s}$. Depicted in Figure 10 is the $\overline{E_{w}}-M$ relationship while keeping all other parameters constant. The mean voltage increases with $\rho u$. This is not surprising; plotting the ${\overline{E_{w}}}^{2}-\rho u$ relationship for $M=1.5$ yields the well-known $n=0.5$ calibration, similar to the one shown in Figure 4 . In addition, $\overline{E_{w}}$ slightly increases with $M$ with a similar slope for all values of $\rho u$. This slope provides an approximate sensitivity coefficient to Mach number

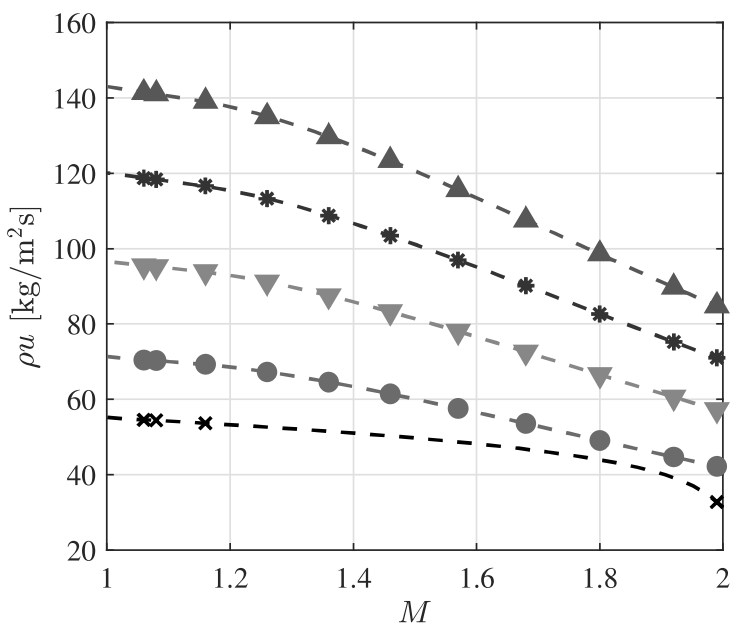

Fig. 7: Theoretical mass flux, $\rho u$, plotted against the Mach number, $M$, for five distinct stagnation pressures, $p_{0}: 0.23$ $(\times), 0.30(\circ), 0.41(\nabla), 0.50(*)$ and $0.60(\triangle)$ atm.

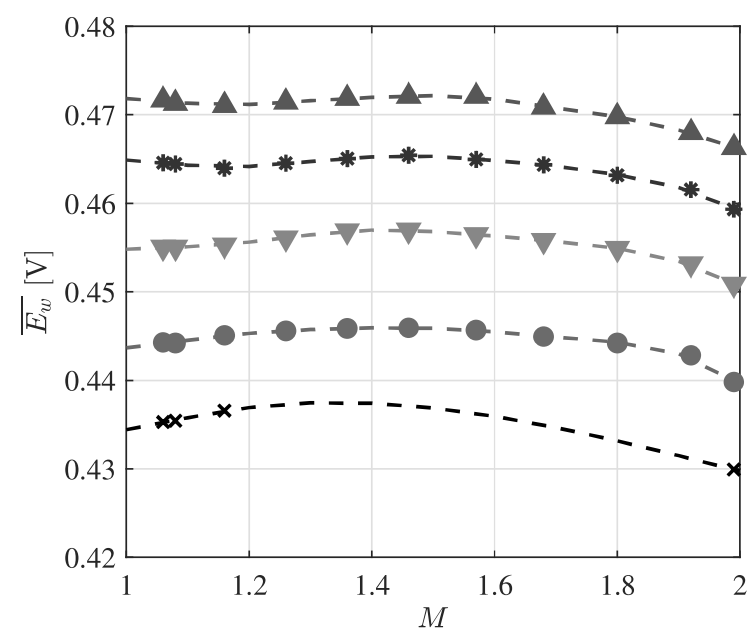

Fig. 8: Mean wire voltage, $\overline{E_{w}}$, plotted against $M$ for five distinct stagnation pressures, $p_{0}: 0.23(\times), 0.30(\circ), 0.41$ $(\nabla), p_{0}=0.50(*)$ and $p_{0}=0.60(\triangle)$ atm.

of $F_{M} \sim O(0.04)$ which points to a non-negligible effect of $M$ on $F_{1}$ in Equation 8, confirming the previous analysis.

According to the analysis presented by Morkovin (1956), $F_{M}$ estimated above depends on both $\partial \eta / \partial M$ and $\partial N u / \partial M$, where $\eta=T_{e} / T_{0}$ is the recovery factor. Therefore, the recovery temperature, $T_{e}$, was measured throughout the data collection process and a systematic increase was measured as the S-NSTAP moved towards the throat. Figure 11 displays an inverse trend between $\eta$ and $M$. Laufer and McClellan (1956) obtained $\eta=0.95 \pm 0.01$ when $R e>20$ and observed a sharp increase in $\eta$ as $R e$ decreased below this value. Depicted in Figure 11, $\eta$ increases from $\eta=0.926$ 


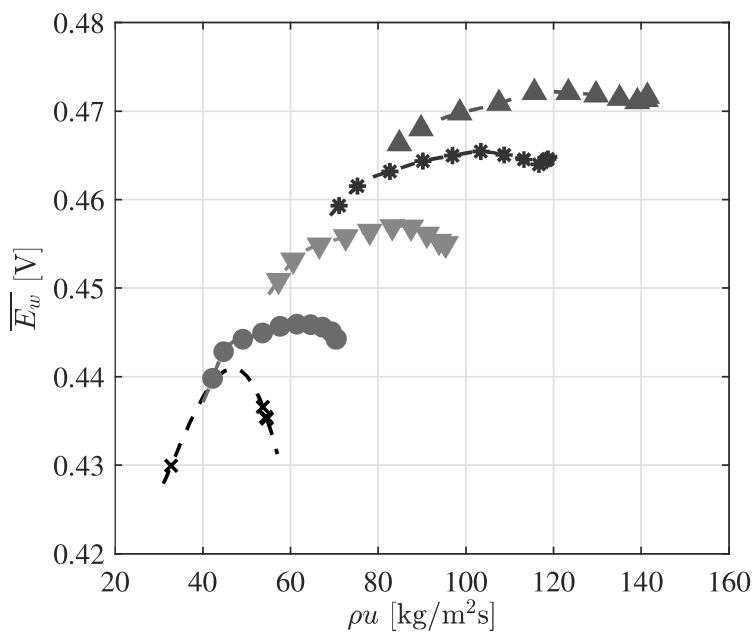

Fig. 9: Mean wire voltage, $\overline{E_{w}}$, plotted against $\rho u$ for five distinct stagnation pressures, $p_{0}: 0.23(\times), 0.30(\circ), 0.41$ $(\nabla), p_{0}=0.50(*)$ and $p_{0}=0.60(\triangle)$ atm.

to 0.967 as $M$ decreases from 2 to 1 , showcasing a similar trend as what was found in Laufer and McClellan (1956). However, since the Reynolds numbers calculated for the SNSTAP were all below 20, slightly larger values of $\eta$ were expected. This discrepancy is likely due to the difference in wire geometry.

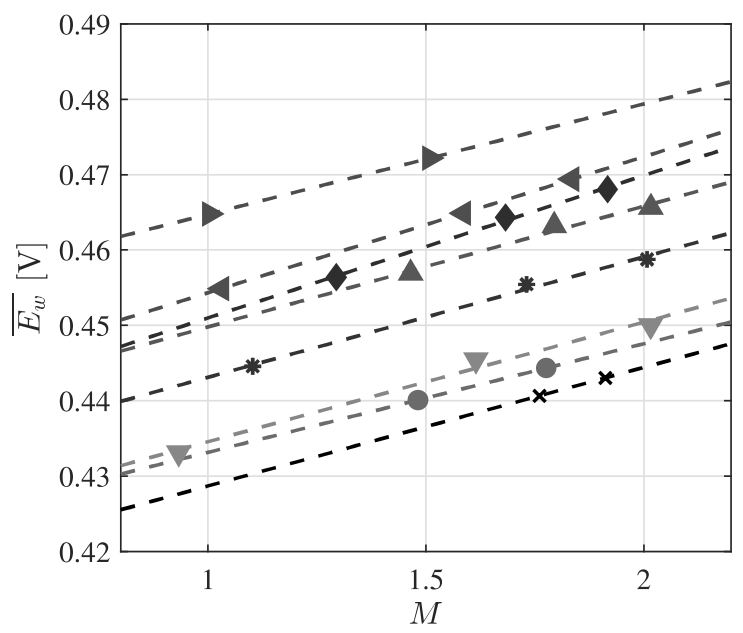

Fig. 10: $\overline{E_{w}}$ versus $M$, keeping all other parameters constant. Data for the following eight values of $\rho u$ were included, in ascending order: $45(\times), 50(\circ), 56(\nabla), 70(*), 83(\triangle), 90$ $(\diamond), 96(\triangleleft)$ and $120(\triangleright) \mathrm{kg} / \mathrm{m}^{2} \mathrm{~s}$.

Knowing the values of $\eta, E_{w}$ and $R_{w}, N u$ was computed and plotted against $M$ for the eight selected values of $\rho u$, the results of which are displayed in Figure 12. Unsurpris-

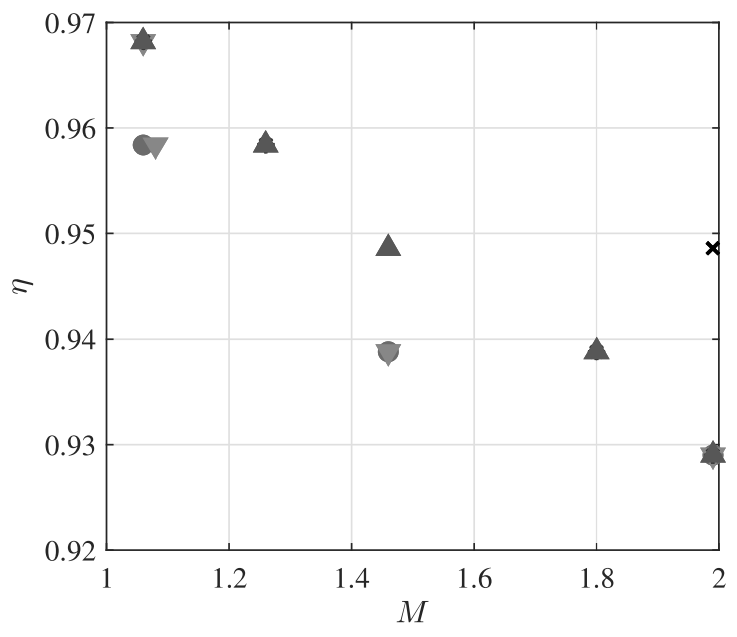

Fig. 11: Recovery ratio, $\eta=\frac{T_{e}}{T_{0}}$, as a function of $M$. Data obtained with the S-NSTAP at five different $p_{0}: 0.23(\times)$, $0.30(\circ), 0.41(\nabla), 0.50(*)$ and $0.60(\triangle)$ atm.

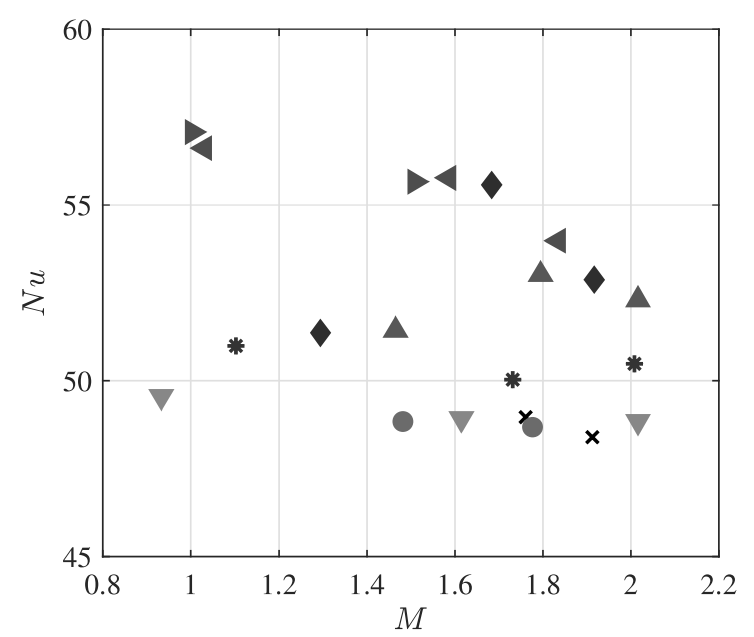

Fig. 12: $N u$ versus $M$, maintaining all other parameters constant. Data for the following eight values of $\rho u$ were included, in ascending order: $45(\times), 50(\circ), 56(\nabla), 70(*)$, $83(\triangle), 90(\diamond), 96(\triangleleft)$ and $120(\triangleright) \mathrm{kg} / \mathrm{m}^{2} \mathrm{~s}$.

ingly, $N u$ increases with $R e$. Contrary to the effect of $\eta$, however, there does not appear to be a systematic trend in the effect of $M$ on the governing normalized heat transfer, i.e. $\partial N u / \partial M \sim 0$. Both facts point to a Mach number sensitivity, $F_{M}$, dependent on the variations of $T_{e}$, according to the aerodynamic conditions $R e$ and $M$. A source of error undoubtedly stems from the estimation of $\eta$ across the nozzle. Due to the low resolution of the acquired $T_{e}$ measurements, this needs to be further investigated. 


\subsection{Effect of overheat ratio}

The measurements presented above were all obtained with a fixed imposed $R_{w}$, leading to an almost constant overheat ratio $\tau$. To shed further light on the effect of overheat ratio on the sensor's heat transfer characteristics, the S-NSTAP was placed in the freestream at $M=2$ and data were collected at four different values of overheat ratio by varying $R_{w}$. It must be noted that, since the measured $R_{w}$ is larger than the sole resistance of the nanoscale sensing element, the true wire temperature is greater than what is estimated using the overheat ratio; this explains why a seemingly low overheat ratio can still lead to sufficient Joule heating.

Although the output voltage increased with an increase in $\tau$, the corresponding $N u$ decreased due to the larger temperature difference, $\Delta T$, between the wire temperature and the recovery temperature. This is depicted in Figure 13, where $\mathrm{Nu}$ is plotted against the square root of the Reynolds number based on the width of the sensing element, $\sqrt{R e_{w t}}$. Lines fit the data well for the three larger values of $\tau$, confirming the square root dependence of $N u$ on $R e_{w t}$, while the data set collected at the lowest $\tau=0.071$ deviates from this linear $N u \sim \sqrt{R e_{w t}}$ trend. Although a decrease of the normalized heat loss $N u$ with $\tau$ was also reported by Kovásznay (1950) and Laufer and McClellan (1956), the physical reasons for this trend remain an open question.

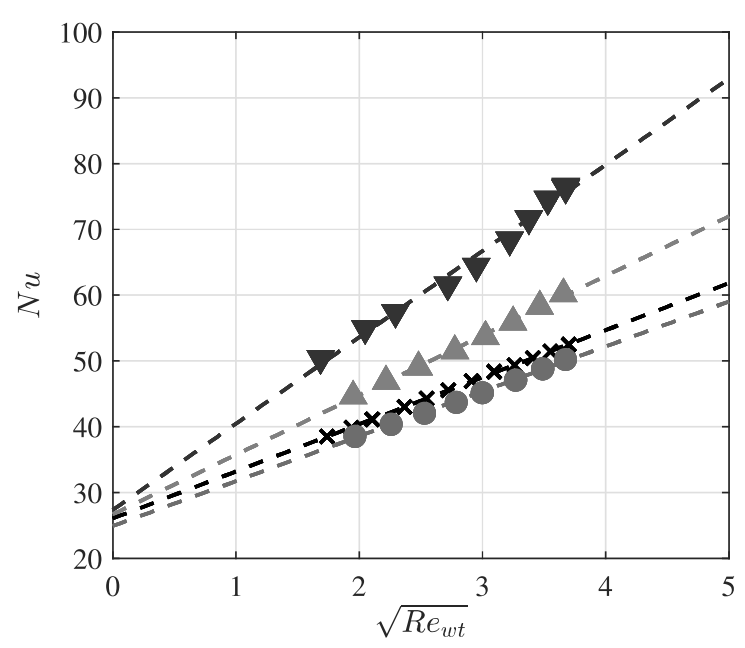

Fig. 13: $N u$ versus $\sqrt{R e_{w t}}$ for four different values of $\tau$ : $0.071(\nabla), 0.24(\triangle), 0.44(\times)$ and $0.56(\circ)$. Linear fits were added to each data set to verify the dependence of $N u$ on $\sqrt{R e_{w t}}$.

\section{Turbulence quantification in a supersonic boundary layer}

Upon characterization of the governing heat transfer mechanisms of the S-NSTAP, an investigation of the spectral content across a $M_{\infty}=2$ boundary layer was undertaken. Figure 14 displays the power spectral density of the measured voltage fluctuations, $\phi_{e}$, without any sort of processing, at both $y / \delta=2.5$ and $y / \delta=0.084$. The wavenumber, $k_{x}$, was computed using Taylor's hypothesis, i.e. $k_{x}=f / u$, where $f$ denotes the frequency and $u$ denotes the streamwise velocity (Smits et al, 2011). As expected, the freestream flow contains much less energy than very close to the wall; visually, a shift of more than a decade is depicted between both spectra. The remarkably clean spectrum at $y / \delta=0.084$ resembles the classical turbulence spectrum (Perry et al, 1986).

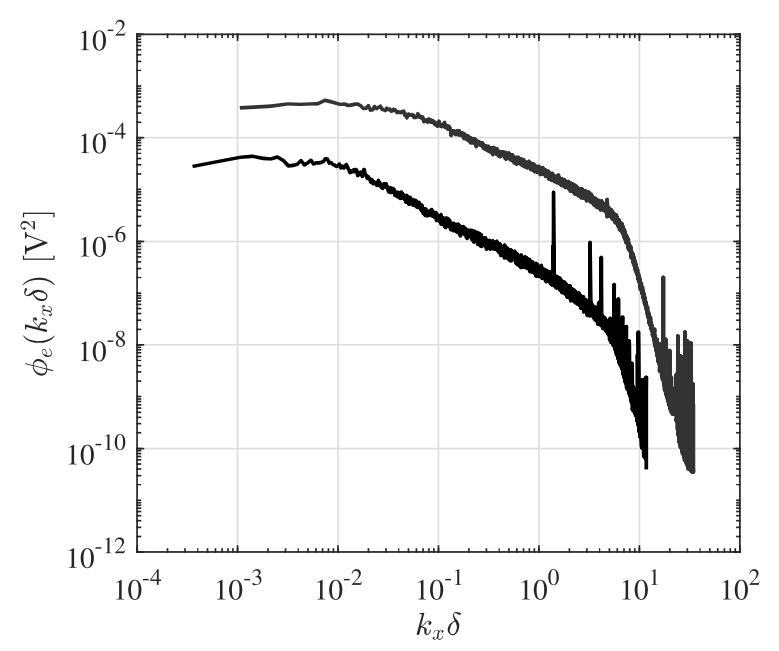

Fig. 14: Power spectral density of unprocessed voltage fluctuations, $\phi_{e}$, as a function of $k_{x} \delta$, computed at $y / \delta=2.5$ (black) and 0.084 (blue). Data collected using the S-NSTAP at $\tau=0.44$ and $p_{0 \infty}=0.5 \mathrm{~atm}$.

In order to further investigate the spectral content present in the supersonic turbulent boundary layer, the calibrated mass flux signals were Fourier transformed. Figure 15 depicts the power spectral density of the streamwise mass flux fluctuations, $\phi_{\rho u}$, the latter of which were normalized by their local mean value, $\overline{\rho u}$. This was done in order to directly compare the HWA to the numerical results from the LES which was performed at a slightly lower $p_{0 \infty}$. Data captured at $y=1 \mathrm{~mm}$ or, equivalently, at $y / \delta=0.086 \pm 0.002$ are plotted. The standard $5 \mu \mathrm{m}$ hot-wire was also calibrated and its normalized power spectral density is included, in black, for comparison. The corresponding non-dimensional wall-normal distance for the LES is $y / \delta=0.09$ where, in this case, $\delta=11.16 \mathrm{~mm}$. Data from the standard hot-wire 
and the LES collapse at the lowest frequencies while the SNSTAP data capture slightly more energy. However, when $k_{x} \delta>0.1$, the S-NSTAP and LES data match well while the conventional hot-wire underpredicts the energy. Interestingly, all three data sets obtained with hot-wire anemometry appear to capture a slight hump around $k_{x} \delta \sim 0.003$ before tailing off. The proximity of the sensor to the end of the converging-diverging nozzle compounded with the relatively low Reynolds number may have led to incomplete development of the turbulent structures. However, this lowfrequency hump may also be attributed to large-scale structures; additional measurements will be performed in order to shed light on the cause of this phenomenon. As of $k_{x} \delta=0.1$, the three spectra obtained via hot-wire anemometry follow the classical $k_{x}^{-1}$ trend for more than a decade (Perry et al, 1986). Moreover, aliasing becomes noticeable in the LES at $k_{x} \delta \sim 5$, where the apparent energetic increase is nonphysical (Jiang et al, 2017). It is clear from these spectra that the S-NSTAP exhibits a superior temporal resolution than that of the conventional hot-wire. A standard square wave test in $M=2$ freestream flow with the use of CTA under a 1:1 bridge configuration resulted in a frequency response after electronic compensation on the order of $300 \mathrm{kHz}$ for the S-NSTAP as opposed to $100 \mathrm{kHz}$ for the standard probe. These values were obtained using the method of Freymuth (1977). By comparing the S-NSTAP's results to previous works, the S-NSTAP measures more turbulence intensity than conventional hot-wires placed in similar flows. The SNSTAP's higher estimated turbulence intensity is likely due to the increased bandwidth of the nanoscale sensor, confirming its increased performance.

Depicted in Figure 16 is the standard deviation of the streamwise mass flux signal throughout the boundary layer. Here, $\sqrt{\overline{(\rho u)^{\prime 2}}}$ is normalized by the local mean mass flux, $\overline{\rho u}$. The results obtained from both the LES and the conventional hot-wire are also included for comparison. All hotwire data shown in Figure 16 were band-pass filtered in order to remove the effect of the large-scale motions; frequencies between $f=2000$ and $100,000 \mathrm{~Hz}$, or equivalently $0.045<f \delta / u_{\infty}<2.25$, contributed to the calculation of the standard deviation, where the freestream streamwise velocity equaled $u_{\infty}=510.8 \mathrm{~m} / \mathrm{s}$. Above $y / \delta \sim 0.4$, all data sets behave similarly and a close agreement is particularly observed between the $p_{0 \infty}=0.5 \mathrm{~atm}$ data set collected with the S-NSTAP and the LES. Below $y / \delta \sim 0.4$ however, the turbulence level obtained with HWA decreases, while it appears to monotonically increase for the LES. This monotonic increase has also been observed using direct numerical simulation (DNS), where the normalized streamwise component of turbulence intensity was plotted as a function of normalized wall-normal distance and a clear maximum in turbulent fluctuations was found at $y / \delta \sim 0.1$ (Martin, 2004).

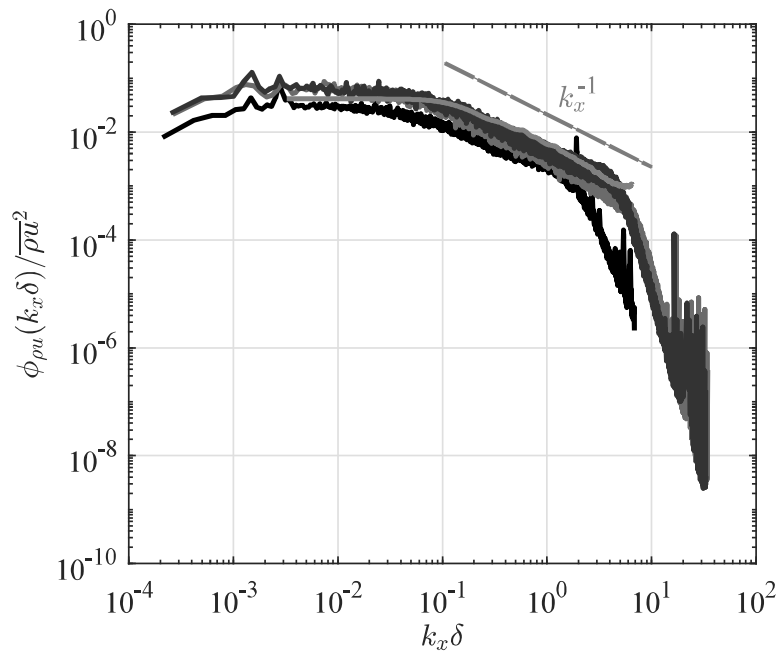

Fig. 15: Power spectral density of the streamwise mass flux fluctuations, $\phi_{\rho u}$, normalized by the corresponding local mean mass flux at a normalized wall-normal distance of $y / \delta=0.086 \pm 0.002$. The S-NSTAP signal collected at $p_{0 \infty}=0.5 \mathrm{~atm}$ is in red while the $p_{0 \infty}=0.8 \mathrm{~atm}$ data set is depicted in blue. The data from the conventional hot-wire, collected at $p_{0 \infty}=0.5 \mathrm{~atm}$, are in black and the LES, performed at $p_{0 \infty}=0.4 \mathrm{~atm}$, is depicted in grey.

This peak in turbulence, observed mid-boundary layer and only captured with HWA, is physical and has been noticed in previous studies. Kistler (1959) traversed a turbulent, zero pressure gradient boundary layer with a $1.27 \mu \mathrm{m}$ diameter hot-wire at three distinct Mach numbers and collected data until $y / \delta=0.1$ in order to prevent Mach number effects from contaminating the data. He observed similar trends for his $M_{\infty}=3.56$ and $M_{\infty}=4.67$ data sets, where a clear peak was found at $y / \delta=0.4$. As for the lowest Mach number data set, $M_{\infty}=1.72$, it appeared to increase monotonically with a decrease in $y / \delta$. Smits et al (1983) later found similar results, where they placed a 2 to $5 \mu \mathrm{m}$ diameter probe in a $M_{\infty}=2.9, R e_{\theta}=77,600$ turbulent boundary layer. A peak of $\overline{\rho u} /(\overline{\rho u})_{\infty} \approx 12 \%$ was found at $y / \delta=0.5$. Lastly, Spina and McGinley (1994) plotted the fluctuating mass flux profile of a $M_{\infty}=11$ helium boundary layer obtained with HWA. A clear peak was also found relatively far from the wall, at $y / \delta=0.5$.

The effects of density, wall-normal velocity and largescale structures were investigated to potentially elucidate this peak in turbulence. As previously stated, hot-wires measure the product of density and velocity while simulation methods, such as LES, allow for the individual examination of streamwise velocity. That being said, the difference in profile throughout the turbulent boundary layer between the streamwise velocity and density could explain the behavior seen in Figure 16. For example, Spina and McGinley 


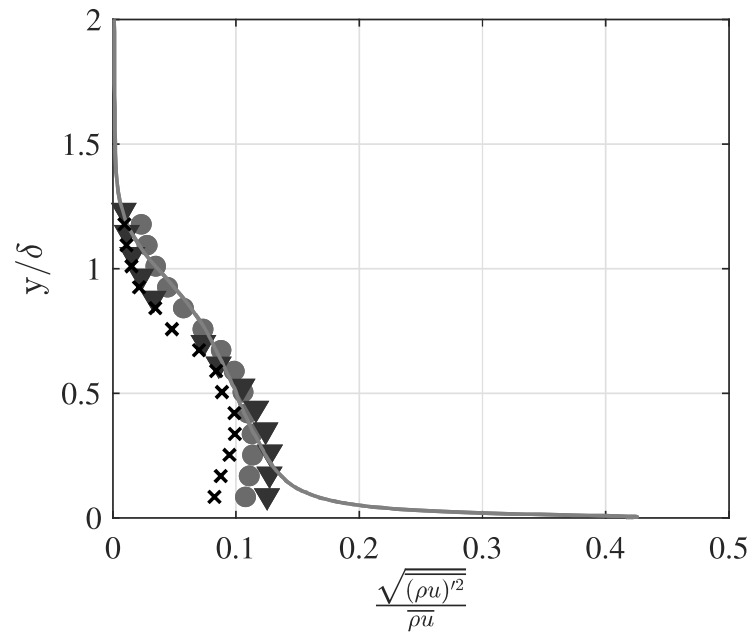

Fig. 16: Standard deviation of the streamwise mass flux signal, $\sqrt{\overline{(\rho u)^{\prime 2}}}$, normalized by the local mean mass flux, $\overline{\rho u}$, across a $M_{\infty}=2$ turbulent boundary layer. Red markers (o) represent S-NSTAP data obtained at $p_{0 \infty}=0.5 \mathrm{~atm}$ while blue markers $(\nabla)$ depict S-NSTAP data obtained at 0.8 atm. The conventional hot-wire data set at $p_{0 \infty}=0.5 \mathrm{~atm}(\times)$ and the LES data set at $p_{0 \infty}=0.4$ atm (grey curve) were also added for comparison.

(1994) attributed their obtained outward shift in peak turbulence to the fact that the density near the wall was much lower than the density farther from the wall $\left(\rho_{\infty} / \rho_{\text {wall }} \approx 35\right)$, a typical phenomenon in hypersonic boundary layers. However, the LES results show a less than twofold variation in density across the $M_{\infty}=2$ boundary layer, invalidating this hypothesis for the present study.

Other than the effect of density, it was hypothesized that the wall-normal velocity fluctuations, $v^{\prime}$, may push the turbulence outwards. Although $v^{\prime}$ was not measured experimentally, data from the LES were used to deter this conjecture; the mean wall-normal mass flux is insignificant compared to its streamwise counterpart, while the turbulence fluctuations in the wall-normal direction are greatest at the wall and decrease as $y / \delta$ increases.

The third and final hypothesis is one regarding largescale structures. Elsinga et al (2010) performed tomographic particle image velocimetry (PIV) to visualize a $M_{\infty}=2.1$, $R e_{\theta}=34,000$ turbulent boundary layer, applied a low-pass filter to the velocity data and noticed the presence of largescale structures farther away from the wall. More recently, Bross et al (2021) performed planar PIV at varying $M$ and $\operatorname{Re}_{\theta}\left(0.3 \leq M \leq 3.0\right.$ and $\left.29,120 \leq \operatorname{Re}_{\theta} \leq 81,180\right)$, compared the superstructures present in both subsonic and supersonic turbulent boundary layers and noticed a $20 \%$ increase in the energetic streamwise wavelengths associated with these large-scale structures. Jiang et al (2017) found a difference, albeit less pronounced, in the low frequency spectral content between their experimental and numerical data. Although the presence of superstructures was considered, their low $R e_{\theta}=4850$ led them to believe that the supersonic flow may be relaminarizing in the divergent segment of the converging-diverging nozzle before entering the test section (Jiang et al, 2017). Although this may have occurred, this must occur systematically in every experiment conducted using different facilities for it to explain the midboundary layer peak in turbulence. Since superstructures have been shown to exist at all Reynolds numbers and their presence in the outer layer of the boundary layer has been suggested, these large-scale structures could be the reason for the peak in variance near $y / \delta=0.5$ (Smits et al, 2011; Kim and Adrian, 1999; Tutkun et al, 2009; Adrian, 2007). Additional measurements will be performed to investigate the presence of large-scale coherent motions and potentially validate this final hypothesis.

\section{Conclusions and outlook}

The purpose of this study was to investigate the response of small-scale thermal sensors in supersonic flows. To achieve this goal, the supersonic variant of the NSTAP, named the S-NSTAP, was tested in a supersonic wind tunnel facility. These measurements allowed for the isolated characterization of the effects of $R e, M$ and $\tau$ on the governing heat transfer of the nanoscale hot-wire. For all tested Mach numbers, a heat transfer calibration of the form $N u \propto \sqrt{R e_{w t}}$ was observed. Contrary to the effect of $R e$, it was found that an increase in $\tau$ led to a decrease in $N u$. A study was also undertaken in order to investigate the effect of $M$ on the governing heat transfer of the S-NSTAP. The increase in $M$ translated to an increase in output voltage, thus pointing to a non-negligible sensitivity of the output to the Mach number. While the effect of $M$ on $N u$ appears to be negligible, the recovery factor $\eta$ is inversely proportional to the Mach number. In addition, the mass flux sensitivity of the S-NSTAP was quantified. Contrary to the $F_{1}=0.25$ coefficient obtained with a conventional $d=5 \mu \mathrm{m}$ cylindrical hot-wire, the S-NSTAP appeared to have a lower sensitivity coefficient of $F_{1}=0.12$. The sensitivity analysis also hints to the existence of a Mach number effect.

The spectral content of the S-NSTAP signal was also investigated from measurements in a $M_{\infty}=2$ turbulent boundary layer. The newly-developed sensor was shown to outperform the conventional hot-wire, exhibiting a frequency response on the order of $300 \mathrm{kHz}$ when operated with a Dantec StreamLine CTA anemometer. Interestingly, the standard deviation of the streamwise mass flux signals did not exhibit a monotonic behavior throughout the boundary layer. Unlike the LES results, the data obtained experimentally showed a peak in the turbulence intensity at approximately $y / \delta=0.4$. 
Applying a band-pass filter on the experimental signals led to a close agreement between all data sets, except very near the wall. The presence of large-scale structures, captured by HWA, may contribute to this discrepancy and must be further explored.

As mentioned, the S-NSTAP exhibited an unparalleled frequency response, rendering it an attractive technique to measure turbulence in supersonic flows. However, certain factors require further investigation. First, the sensor follows the classical King's law, while revealing Knudsen numbers in the range $0.25<K n_{w t}<1$. It is of interest to inquire to what extent rarefied gas dynamics affect the governing heat transfer mechanisms at play. Numerical simulations covering slip flow and free-molecule flow regimes would be of great interest to shed light on this question. In addition, the impact of the sensor's geometry should be further investigated, specifically the stub geometry supporting the sensing element and its effect on the heat transfer output. Finally, the thermal response of the nanoscale probe remains to be quantified by performing step changes in the traversing current in an electronic circuit without compensation.

Acknowledgements The authors warmly thank Alejandro Gómez Mesa for helping with the compilation of the heat transfer results. Prof. Lionel Larchevêque is also thanked for sharing the results of his numerical simulation. The assistance of Kelly Huang and Alexander Piqué in the sensor manufacturing is gratefully acknowledged. The financial support from the Labex Mécanique is gratefully acknowledged. Lastly, the authors kindly acknowledge the support of the Wenner-Gren Foundations.

\section{References}

Adrian RJ (2007) Hairpin vortex organization in wall turbulence. Physics of Fluids 19:041,301

Bailey SCC, Kunkel GJ, Hultmark M, Vallikivi M, Hill JP, Meyer KA, Tsay C, Arnold CB, Smits AJ (2010) Turbulence measurements using a nanoscale thermal anemometry probe. Journal of Fluid Mechanics 663:160-179

Baldwin LV, Sandborn VA, Laurence JC (1960) Heat transfer from transverse and yawed cylinders in continuum, slip, and free molecule air flows. Journal of Heat Transfer pp 77-86

Barre S, Dupont P, Dussauge JP (1992) Hot-wire measurements in turbulent transonic flows. European Journal of Mechanics-B/Fluids 11(4):439-454

Bross M, Scharnowski S, Kähler CJ (2021) Large-scale coherent structures in compressible turbulent boundary layers. Journal of Fluid Mechanics 911

Comte-Bellot G (1976) Hot-wire anemometry. Annual Review of Fluid Mechanics 8:209-231

Dewey Jr CF (1961) Hot-wire measurements in low Reynolds number hypersonic flows. Tech. rep., Guggen- heim Aeronautical Laboratory California Institute of Technology

Dewey Jr CF (1965) A correlation of convective heat transfer and recovery temperature data for cylinders in compressible flow. International Journal of Heat and Mass Transfer 8(2):245-252

Dupont P, Debiève JF (1992) A hot wire method for measuring turbulence in transonic or supersonic heated flows. Experiments in fluids 13:84-90

Elsinga GE, Adrian RJ, Van Oudheusden BW, Scarano F (2010) Three-dimensional vortex organization in a highReynolds-number supersonic turbulent boundary layer. Journal of Fluid Mechanics 644:35-60

Fan Y, Arwatz G, Van Buren TW, Hoffman DE, Hultmark M (2015) Nanoscale sensing devices for turbulence measurements. Experiments in Fluids 56:138

Freymuth P (1977) Frequency response and electronic testing for constant-temperature hot-wire anemometers. Journal of Physics E: Scientific Instruments 10(7):705

Gatski TB, Bonnet JP (2009) Compressibility, turbulence and high speed flow. Elsevier

Jiang T, Schreyer AM, Larchevêque L, Piponniau S, Dupont P (2017) Velocity spectrum estimation in shockwave/turbulent boundary-layer interaction. AIAA Journal 55(10):3486-3498

Kim KC, Adrian RJ (1999) Very large-scale motion in the outer layer. Physics of Fluids 11(2):417-422

King LV (1914) XII. On the convection of heat from small cylinders in a stream of fluid: Determination of the convection constants of small platinum wires with applications to hot-wire anemometry. Phil Trans R Soc Lond A 214(509-522):373-432

Kistler AL (1959) Fluctuation measurements in a supersonic turbulent boundary layer. The Physics of Fluids 2(3):290296

Kokmanian K (2020) Development of a nanoscale hotwire probe for supersonic flow applications. $\mathrm{PhD}$ thesis, Princeton University

Kokmanian K, Scharnowski S, Bross M, Duvvuri S, Fu MK, Kähler CJ, Hultmark M (2019) Development of a nanoscale hot-wire probe for supersonic flow applications. Experiments in Fluids 60:150

Kovásznay LSG (1950) The hot-wire anemometer in supersonic flow. Journal of the Aeronautical Sciences 17(9):565-572

Kovásznay LSG, Törmarck SIA (1950) Heat loss of hotwires in supersonic flow. Tech. Rep. 127, The Johns Hopkins University

Laufer J, McClellan R (1956) Measurements of heat transfer from fine wires in supersonic flows. Journal of Fluid Mechanics 1(3):276-289

Martin MP (2004) DNS of hypersonic turbulent boundary layers. In: Proceedings of the 34th AIAA Fluid Dynamics 
Conference and Exhibit, p 2337

Morkovin MV (1956) Fluctuations and hot-wire anemometry in compressible flows. Tech. Rep. AGARDograph 24, North Atlantic Treaty Organization, Advisory Group for Aeronautical Research and Development

Perry AE, Henbest S, Chong MS (1986) A theoretical and experimental study of wall turbulence. Journal of Fluid Mechanics 165:163-199

Rapp BE (2017) Microfluidics: Modeling, Mechanics and Mathematics. Elsevier

Schreyer AM, Larchevêque L, Dupont P (2016) Method for spectra estimation from high-speed experimental data. AIAA Journal 54(2):557-568

Smits AJ, Hayakawa K, Muck KC (1983) Constant temperature hot-wire anemometer practice in supersonic flows. Experiments in Fluids 1:83-92

Smits AJ, McKeon BJ, Marusic I (2011) High-Reynolds number wall turbulence. Annual Review of Fluid Mechanics 43

Spina EF, McGinley CB (1994) Constant-temperature anemometry in hypersonic flow: critical issues and sample results. Experiments in Fluids 17:365-374

Stalder JR, Goodwin G, Creager MO (1951) A comparison of theory and experiment for high-speed free-molecule flow. Tech. Rep. 1032, National Advisory Committee for Aeronautics

Stalder JR, Goodwin G, Creager MO (1952) Heat transfer to bodies in a high-speed rarified-gas stream. Tech. Rep. 1093, National Advisory Committee for Aeronautics

Tropea C, Yarin AL, Foss JF (2007) Springer handbook of experimental fluid mechanics. Springer

Tutkun M, George WK, Delville J, Stanislas M, Johansson PBV, Foucaut JM, Coudert S (2009) Two-point correlations in high Reynolds number flat plate turbulent boundary layers. Journal of Turbulence 10(21):1-23

Weltmann RN, Kuhns PW (1960) Heat transfer to cylinders in crossflow in hypersonic rarefied gas streams. National Aeronautics and Space Administration 